\title{
Primary Isolated Case of Drug Induced Pancreatitis Misdiagnosed as Idiopathic Pancreatitis
}

\author{
Ali Raza Ghani1 ${ }^{*}$, Kanwal Saba², Mulazzim Hussain ${ }^{3}$, Amjad G. Sheikh4 \\ ${ }^{1}$ Thomas Jefferson University Hospital, Philadelphia, USA \\ ${ }^{2}$ Department of Pathology, King Edward Medical University, Lahore, Pakistan \\ ${ }^{3}$ Department of Pathology, School of Medicine, American University of Barbados, Wildey, Barbados \\ ${ }^{4}$ Medicine \& Pediatrics Carthage Area Hospital, Carthage, USA \\ Email: ${ }^{*}$ alighani152@gmail.com, asheikhmd@cahny.org
}

Received 12 April 2014; revised 11 May 2014; accepted 10 June 2014

Copyright (C) 2014 by authors and Scientific Research Publishing Inc.

This work is licensed under the Creative Commons Attribution International License (CC BY).

http://creativecommons.org/licenses/by/4.0/

c) (i) Open Access

\begin{abstract}
Drug Induced Pancreatitis is a very common cause of acute pancreatitis in older patients and is often treated by just symptomatic treatment. An old hypertensive lady was admitted repeatedly to emergency department and treated as Idiopathic Pancreatitis. Initial labs showed elevated pancreatic enzymes. Detailed history showed that increase in thiazides dosage was the cause of her pancreatitis. It was later diagnosed as a case of thiazide induced pancreatitis. It is important to consider medication as a cause of acute pancreatitis in especially in older people presenting with acute pancreatitis without common risk factors.
\end{abstract}

\section{Keywords}

Acute Pancreatitis, Diagnosis, CT Scan, Gastroscopy, Thiazides, Hypertension, Lipase, ERCP

\section{Introduction}

Pancreatitis is inflammation of the pancreas, an organ that produces several enzymes to aid in the digestion of food, as well as the hormone insulin, which controls the level of sugar (glucose) in the blood. The pancreas is located in the upper abdomen, behind the stomach. It is affected broadly by many risk factors and medications are very common causes of pancreatitis. Among adverse drug reactions, pancreatitis is often-ignored because of the difficulty in implicating a drug as its cause. The physician should have a high index of suspicion for DIP,

${ }^{*}$ Corresponding author.

How to cite this paper: Ghani, A.R., Saba, K., Hussain, M. and Sheikh, A.G. (2014) Primary Isolated Case of Drug Induced Pancreatitis Misdiagnosed as Idiopathic Pancreatitis. Case Reports in Clinical Medicine, 3, 416-419.

http://dx.doi.org/10.4236/crcm.2014.37092 
especially in specific subpopulations such as geriatric patients who may be on multiple medications, HIV+ patients, cancer patients, and patients receiving immune modulatory agents. In this report, we describe a case of thiazide induced pancreatitis which was treated as Idiopathic pancreatitis [1]-[4].

Although thiazide drugs are increasingly being used in clinical practice, data on incidence of acute pancreatitis with this class of drug are lacking. We evaluated the extent of this adverse effect in patients on thiazide on our follow-up.

\section{Case Report}

A 79-year-old lady with history of hypertension, presented to Carthage Hospital Emergency with headache, nausea vomiting and uncontrolled hypertension. She was treated with antihypertensive medications and her blood pressure improved with treatment. After 4 days, she presented with intermittent epigastric pain, non-radiating for past 3 days associated with nausea, vomiting. She also reported dark brown stools in last few days. She had 4 episodes of vomiting the day before admission. There was no history of ethanol abuse, trauma, steroid use, gallstones, and melena. On physical examination, epigastric tenderness was present but rest of physical exam was normal. She was admitted to hospital with diagnosis of acute pancreatitis. She was kept NPO and given IV fluids and morphine to control pain.

Labs: Sodium 144, Potassium 3.7, Chloride 108, Bicarbonate 26, Anion Gap 10, Calcium 8.4, BUN 15, Creatinine 0.8, Albumin 3.3, Total Protein 5.9, ALT 100, AST 113, Triglycerides 174, Total Cholesterol 137, Lipase level of 1552.

CT of abdomen showed mild peri pancreatic inflammatory stranding, trace free fluid and hazy cortical margins at head and uncinate process and $1.1 \times 1 \mathrm{~cm}$ hypodensity in the neck of pancreas. Therefore surgical service advised for CT abdomen with or without contrast. Gastroenterology was consulted who proposed getting CT abdomen with contrast with pancreatic protocol which showed non enhancing $1 \mathrm{~cm}$ cystic lesion within the pancreatic neck. Ultrasound showed no evidence of acute cholecystitis. An ERCP was also normal.

When the patient came back to Carthage Hospital for routine follow up, she was still having repeated attacks of acute pancreatitis. The detailed history revealed that her thiazide medication dosage was increased because of admission to ED due to uncontrolled hypertension. So after ruling out all the other causes, her thiazides were discontinued and her attacks of pancreatitis were resolved and patient improved.

\section{Discussion}

This case illustrates the incidence of severe pancreatitis in even asymptomatic patients who are on combination antihypertensive medications especially older people who had their doses increased to control hypertension. So I would suggest that clinicians should be very careful when they adjust doses of thiazides in old patients. It is a very well-known fact which is often undermined in many people presenting to emergencies. Reports of drug-induced acute pancreatitis (AP) have been published since the 1950s, and each year the list of drugs associated with AP increases. There are many etiological risk factors for AP, including a history of alcohol abuse, gallstones, endoscopic retrograde cholangiopancreatography and manometry, trauma or surgical procedures near the pancreas, certain medications, hyperlipidemia, infection, and chronic hypercalcemia [5]. Knowledge of the true incidence of drug-induced AP is dependent on clinicians excluding other possible causes and reporting the event. It can be difficult to rule out other causes of AP, especially in patients who have multiple comorbidities, use multiple medications, and have potentially unknown underlying risk factors. A retrospective study conducted in Germany concluded that the incidence of drug-induced AP is $1.4 \%$. A national survey performed in Japan in 1999 reported that 1.2\% of all cases of AP were drug induced. Drug-induced AP is rare but should not be overlooked in a patient who presents with idiopathic AP [6]-[8].

When AP progresses to a severe illness, patients can experience extended hospital stays and increased health care costs. Neoptolemos and colleagues reported that approximately $25 \%$ of patients who develop AP will require intensive care treatment. In a retrospective study conducted between January 1992 and December 1996, patients with AP who required intensive care treatment had an average intensive care unit stay of 9 days and an average total hospital stay of 39 days. The investigators also concluded that the average overall hospital cost was $\$ 96,891$. Fortunately, AP patients typically regain complete functional ability and can return to employment and other normal activities [9] [10].

In previous years, experts created a classification system that addressed the likelihood that certain drugs 
would be associated with AP, using the categories of definite, probable, and questionable/possible. Over the years, the list of medications associated with AP has increased. Most recently, Badalov and colleagues expanded the classification system to five categories: Ia, Ib, II, III, and IV [11] [12].

The mechanisms of action for drug-induced AP are based on theories extracted from case reports, case-control studies, animal studies, and other experimental data. In general, some potential mechanisms of action for drug-induced AP include pancreatic duct constriction, cytotoxic and metabolic effects, accumulation of a toxic metabolite or intermediary, and hypersensitivity reactions [12].

Published case reports of drug-induced AP exist for at least 40 drugs of the top 200 most prescribed medications. This article reviews data on five drug classes or drugs commonly associated with AP: 3-hydroxy-3-methyl glutaryl coenzyme A (HMG-CoA) reductase inhibitors, ACE inhibitors, estrogens/HRT, diuretics, highly active antiretroviral therapy (HAART), and valproic acid. Most of these drugs are among the top 200 most prescribed medications for 2007 [13].

In this observational study, we noted one patient with acute pancreatitis, which was mild and self-limiting on drug withdrawal.

Laboratory investigation of this patient was in favor or acute pancreatitis especially high level of Lipase. Gastroenterology was consulted who proposed getting CT abdomen with contrast with pancreatic protocol which showed non enhancing $1 \mathrm{~cm}$ cystic lesion within the pancreatic neck. Ultrasound showed no evidence of acute cholecystitis. In this case, an ERCP was also normal. The possible reason in this case was ruled out on history when patient came back to Hospital for routine follow up as she was still having repeated attacks of acute pancreatitis. The history revealed that high dosages of thizides were the cause of this recurrent pancreatitis, the dose of thiazides was increased due to poorly controlled blood pressure at lower dose. So after ruling out all the other causes, her thiazides were discontinued and her attacks of pancreatitis were resolved and patient improved.

Drug induced AP should be kept on differential list specially when patient is on multiple drug therapy. Laboratory tests, gastroscopy and imaging can be challenging and should be analyzed thoroughly supplementing with other data where necessary. History plays an important role in the differrential diagnosis of such cases. Larger prospective database with a hypertensive and nonhypertensive control population is likely to shed more light on this issue.

\section{Conclusion}

Acute pancreatitis is well known to occur at a greater frequency in hypertensive patients with high dosage of thiazides therapy. The heightened risk of this dreaded disease is suspected with the use of high dosage of thiazides. In this small study with its limitations, we did not note any greater risk of acute pancreatitis than expected in a cohort of hypertensive patients. However, greater watchfulness for this dreaded adverse effect and avoidance of its high dosages for pancreatitis is an initial approach to save the patient from this dreadful disease.

\section{Conflict of Interest}

All authors have no any actual or potential conflict of interest including any financial, personal or other relationships with other people or organizations within three years of beginning the submitted work that could inappropriately influence, or be perceived to influence, our work.

\section{Submission Declaration and Verification}

We also declare that the work described has not been published previously except in the form of an abstract or as part of a published lecture or academic thesis or as an electronic preprint, is approved that, if accepted, it will not be published elsewhere in the same form, in English or in any other language, including electronically without the written consent of the copyright-holder.

\section{Authorship}

Ali Raza Ghani was the principal researcher and prepared the first draft of the report, KS was co-supervisor, Mulazim Hussain Bukhari, was supervisor. Amjad G. Sheikh reviewed the article. 


\section{References}

[1] IAP/APA (2013) Evidence-Based Guidelines for the Management of Acute Pancreatitis. Pancreatology: Official Journal of the International Association of Pancreatology (IAP), 13, e1-e15.

[2] Johnstone, M., Marriott, P., Royle, T.J., Richardson, C.E., Torrance, A., Hepburn, E., et al. (2014) The Impact of Timing of Cholecystectomy Following Gallstone Pancreatitis. The Surgeon: Journal of the Royal Colleges of Surgeons of Edinburgh and Ireland, 12, 134-140.

[3] Tenner, S., Baillie, J., DeWitt, J. and Vege, S.S. (2013) American College of Gastroenterology Guideline: Management of Acute Pancreatitis. The American Journal of Gastroenterology, 108, 1400-1416.

[4] Kim, D.B., Cho, Y.K., Song, H.J. and Song, B.C. (2013) A Case of Acute Pancreatitis and Acute Hepatitis Caused by Ingestion of Ceramiumkondoi. The Korean Journal of Gastroenterology, 62, 306-309.

[5] Sekimoto, M., Takada, T., Kawarada, Y., Hirata, K., Mayumi, T., Yoshida, M., Hirota, M., Kimura, Y., Takeda, K., Isaji, S., et al. (2006) JPN Guidelines for the Management of Acute Pancreatitis: Epidemiology, Etiology, Natural History, and Outcome Predictors in Acute Pancreatitis. Journal of Hepato-Biliary-Pancreatic Surgery, 13, 10-24. http://dx.doi.org/10.1007/s00534-005-1047-3

[6] Vinklerova, I., Prochazka, M., Prochazka, V. and Urbanek, K. (2010) Incidence, Severity, and Etiology of Drug-Induced Acute Pancreatitis. Digestive Diseases and Sciences, 55, 2977-2981. http://dx.doi.org/10.1007/s10620-010-1277-3

[7] Lankisch, P.G., Droge, M. and Gottesleben, F. (1995) Drug Induced Acute Pancreatitis: Incidence and Severity. Gut, 37, 565-567. http://dx.doi.org/10.1136/gut.37.4.565

[8] Garg, R., Chen, W. and Pendergrass, M. (2010) Acute Pancreatitis in Type 2 Diabetes Treated with Exenatide or Sitagliptin: A Retrospective Observational Pharmacy Claims Analysis. Diabetes Care, 33, 2349-2354. http://dx.doi.org/10.2337/dc10-0482

[9] Neoptolemos, J.P., Raraty, M., Finch, M. and Sutton, R. (1998) Acute Pancreatitis: The Substantial Human and Financial Costs. Gut, 42, 886-891. http://dx.doi.org/10.1136/gut.42.6.886

[10] Soran, A., Chelluri, L., Lee, K.K. and Tisherman, S.A. (2000) Outcome and Quality of Life of Patients with Acute Pancreatitis Requiring Intensive Care. The Journal of Surgical Research, 91, 89-94. http://dx.doi.org/10.1006/jsre.2000.5925

[11] Badalov, N., Baradarian, R., Iswara, K., Li, J., Steinberg, W. and Tenner, S. (2007) Drug-Induced Acute Pancreatitis: An Evidence-Based Review. Clinical Gastroenterology and Hepatology: The Official Clinical Practice Journal of the American Gastroenterological Association, 5, 648-661.

[12] Engel, T., Justo, D., Amitai, M., Volchek, Y. and Mayan, H. (2013) Nilotinib-Associated Acute Pancreatitis. The Annals of Pharmacotherapy, 47, e3. http://dx.doi.org/10.1345/aph.1R334

[13] Underwood, T.W. and Frye, C.B. (1993) Drug-Induced Pancreatitis. Clinical Pharmacy, 12, 440-448. 
Scientific Research Publishing (SCIRP) is one of the largest Open Access journal publishers. It is currently publishing more than 200 open access, online, peer-reviewed journals covering a wide range of academic disciplines. SCIRP serves the worldwide academic communities and contributes to the progress and application of science with its publication.

Other selected journals from SCIRP are listed as below. Submit your manuscript to us via either submit@scirp.org or Online Submission Portal.
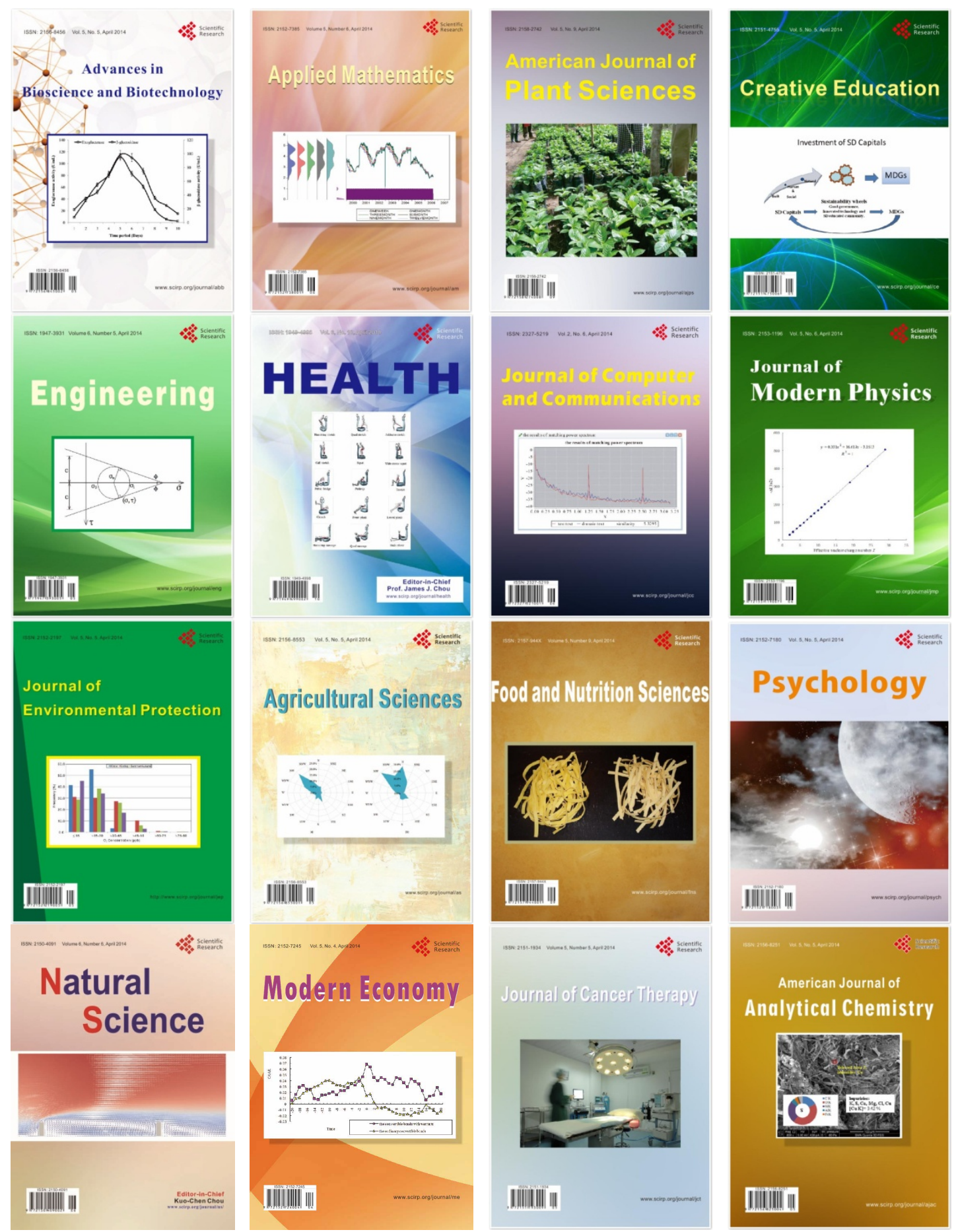